\title{
Breakdown of continuum mechanics for nanometre-wavelength rippling of graphene
}

\author{
Levente Tapasztó ${ }^{1 \star}$, Traian Dumitrică ${ }^{2}$, Sung Jin $\mathrm{Kim}^{3}$, Péter Nemes-Incze ${ }^{1}$, Chanyong Hwang ${ }^{3}$ \\ and László P. Biró
}

\begin{abstract}
Understanding how the mechanical behaviour of materials deviates at the nanoscale from the macroscopically established concepts is a key challenge of particular importance for graphene, given the complex interplay between its nanoscale morphology and electronic properties ${ }^{1-5}$. In this work, the (sub)nanometre-wavelength periodic rippling of suspended graphene nanomembranes has been realized by thermal strain engineering and investigated using scanning tunnelling microscopy. This allows us to explore the rippling of a crystalline membrane with wavelengths comparable to its lattice constant. The observed nanorippling mode violates the predictions of the continuum model ${ }^{6}$, and evidences the breakdown of the plate idealization ${ }^{7}$ of the graphene monolayer. Nevertheless, microscopic simulations based on a quantum mechanical description of the chemical binding accurately describe the observed rippling mode and elucidate the origin of the continuum model breakdown. Spatially resolved tunnelling spectroscopy measurements indicate a substantial influence of the nanoripples on the local electronic structure of graphene and reveal the formation of onedimensional electronic superlattices.
\end{abstract}

The nanoscale landscape is expected to markedly influence the electronic properties of the ideally two-dimensional graphene membranes ${ }^{8-10}$. When the local curvature of the graphene sheet is of the nanometre scale, the electronic structure is substantially modified by altering the $\pi$-orbital energy $\left(\sigma-\pi\right.$ rehybridization $\left.^{7}\right)$ and modifying the nearest-neighbour hopping integrals, which can induce a local shift of the electrochemical potential ${ }^{9}$ as well as give rise to large pseudo-magnetic fields ${ }^{3,4}$. In fact, random nanoscale wrinkles are intrinsic to graphen $\mathrm{e}^{11}$, inducing spatially disordered charge density fluctuations, which act as source of disorder for the propagating charge carriers ${ }^{12,13}$. In contrast, periodic ripples of nanometre wavelength-if achieved - comprise a much richer physics, giving rise to electronic superlattices ${ }^{9}$, which fundamentally alter the quasi-particle energy dispersion and can render the propagation velocity of the charge carriers highly anisotropic ${ }^{14}$. Furthermore, it has been predicted that periodic graphene ripples can be employed for opening a (pseudo-) bandgap ${ }^{3,9}$, which could eliminate the lithographic patterning of graphene and thus the problem of edge quality.

Engineering of micrometre-scale periodic ripples in suspended graphene has been experimentally demonstrated ${ }^{6}$ with wavelengths ranging from a few hundred nanometres up to several micrometres. In the reported rippling regime the results were found to follow the predictions of the continuum plate mechanics quite accurately $y^{6}$.
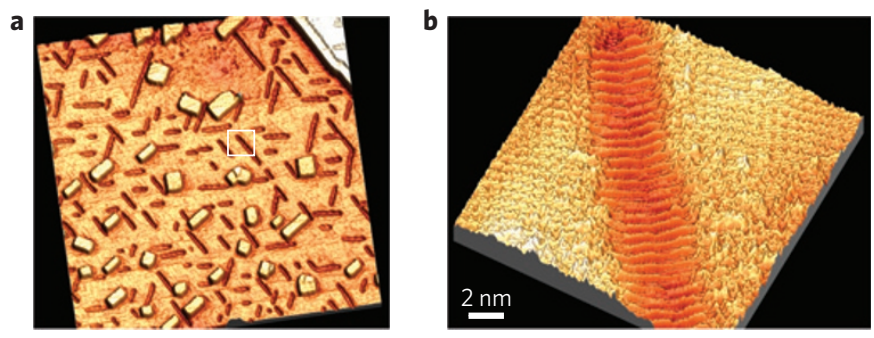

Figure 1 | Three-dimensional STM images of nanotrenches and graphene nanoripples. a, STM image $(300 \times 300 \mathrm{~nm})$ of a reconstructed $\mathrm{Cu}(111)$ surface continuously covered by graphene. The rectangular protrusions are single-atom-height $\mathrm{Cu}$ adatom clusters, whereas the trench-like features correspond to vacancy islands with well-defined widths of $5 \mathrm{~nm}$ and oriented along three particular directions. $\mathbf{b}$, High-resolution STM image of a nanotrench revealing the nanoscale periodic rippling of the graphene membrane suspended over the trench.

To exploit the nanomechanical characteristics of graphene, it is of particular interest to explore down to which length scale the classical membrane behaviour of graphene persists, and how it is altered below that. Furthermore, as the influence of ripples on the electronic structure, as well as the chemical reactivity ${ }^{15}$, of graphene is expected to markedly increase with the local curvature $^{8}$, downscaling the ripple wavelength to the nanometre regime is also of high practical relevance. To implement the nanoscale periodic rippling of graphene, one can employ the strain engineering of nanometre-sized suspended graphene membranes, as the rippling wavelength should scale with the square root of the membrane dimensions ${ }^{16}$

Here, we show that such suspended graphene nanomembranes naturally form during the chemical vapour deposition growth of graphene on $\mathrm{Cu}(111)$ single crystals. The scanning tunnelling microscopy (STM) investigation of the as-grown samples revealed the presence of reconstructed $\mathrm{Cu}$ surface areas underneath the continuous graphene layer. These reconstructions of the $\mathrm{Cu}(111)$ surface consist of monolayer-height $\mathrm{Cu}$ adatom clusters as well as trench-like vacancy islands (see Fig. 1a). Similar reconstructions have been previously reported on single-crystalline $\mathrm{Cu}$ and $\mathrm{Ag}$ surfaces $^{17,18}$. For more details concerning the sample preparation and the origin of the surface reconstructions, see Supplementary Information. The trench-like vacancy islands have well-defined widths of about $5 \mathrm{~nm}$ and their length is typically in the range of $20-80 \mathrm{~nm}$. The atomic-resolution topographic STM images

\footnotetext{
${ }^{1}$ Institute for Technical Physics and Materials Science, Research Centre for Natural Sciences, H-1525 Budapest, Hungary, ${ }^{2}$ Department of Mechanical Engineering, University of Minnesota, Minneapolis, Minnesota 55455, USA, ${ }^{3}$ Center for Nano-imaging Technology, Korea Research Institute of Standards and Science, 267 Gajeong-Ro, Yuseong, Daejon 305-340, Korea. *e-mail: tapaszto.levente@ttk.mta.hu.
} 
reveal the honeycomb lattice of graphene all over the sample surface, including the adatom pads and nanotrenches, with these latter features providing ribbon-like areas of suspended graphene nanomembranes. As evidenced from high-resolution STM measurements, these nanomembranes exhibit highly regular periodic oscillations, with a wavelength of $\sim 0.7 \mathrm{~nm}$ and modulation of $\sim 0.1 \mathrm{~nm}$ superposed on the honeycomb atomic lattice of graphene (Figs $1 \mathrm{~b}$ and 2). The reported nanorippling mode (with $\lambda=0.7 \pm 0.1 \mathrm{~nm}, A=0.5 \pm 0.2 \AA$ ) has been observed on all of the more than thirty trenches investigated. Periodic oscillations in the STM images of graphene can often be attributed to electron interference ${ }^{19}$. Owing to their nature, these interference patterns are expected to strongly depend on the bias voltage (energy) used for imaging ${ }^{20}$. However, in our case, the experimentally observed pattern was practically unchanged (apart from a slight variation of the amplitude) on varying the bias voltage. This is a clear signature of its topographic (structural) origin. We could also exclude the possibility that the observed features are Moiré patterns ${ }^{2,18}$, as the graphene sheet is not in direct contact with the substrate above the trenches (for direct evidence, see Supplementary Information). We propose the following mechanism for the formation of the graphene nanoripples. During graphene growth, at temperatures of about $1,000{ }^{\circ} \mathrm{C}$ (ref. 21), a massive migration of the surface $\mathrm{Cu}$ atoms takes place underneath the growing graphene $\mathrm{e}^{22}$. This gives rise to the observed surface reconstructions including the nanotrenches. On cooling the sample from the growth $\left(\sim 1,000{ }^{\circ} \mathrm{C}\right)$ to room temperature, the $\mathrm{Cu}$ substrate contracts, whereas the overgrown graphene layer tries to expand according to its negative thermal expansion coefficient ${ }^{6}$. Consequently, where graphene is in direct contact, that is, pinned to the $\mathrm{Cu}$ substrate, it is subjected to a strong compressive stress as also suggested by previous Raman investigations ${ }^{23}$. However, in those areas where the graphene is suspended above the nanotrenches it is allowed to relax some amount of the compressive strain through out-of-plane deformation (rippling). The amount of strain due to the cooling to room temperature can be approximated by: $\varepsilon \approx \Delta T\left(\alpha_{\mathrm{Cu}}-\alpha_{\mathrm{Gr}}\right)$, where $\Delta T$ is the temperature difference between $1,000^{\circ} \mathrm{C}$ and ambient, and $\alpha_{\mathrm{Cu}}=16.6 \times 10^{-6} \mathrm{~K}^{-1}$ and $\alpha_{\mathrm{Gr}}=-7 \times 10^{-6} \mathrm{~K}^{-1}$ are the thermal expansion coefficient for $\mathrm{Cu}$ and graphene, respectively. This formula estimates the emerging compressive strain in the asgrown graphene samples to $\varepsilon=2.2 \%$. Here we note that this is only a rough estimate as the thermal expansion coefficient of graphene is also expected to be temperature dependent ${ }^{24}$. The strain can also be experimentally estimated from measuring the surface length of the graphene ripples ${ }^{23}$, yielding similar strain values of a few per cent. This thermally induced strain is responsible for the formation of the observed nanoripples in the suspended graphene areas.

As the curvature of a crystalline membrane becomes commensurate with the lattice constant, the applicability of continuum mechanics becomes uncertain. To verify whether the classical behaviour of graphene observed for micrometre-scale ripples ${ }^{6}$ remains valid also at the nanoscale, we attempted to interpret the observed graphene nanoripples within the established framework of continuum mechanics. The periodic ripples developed in a compressed thin plate of thickness $t$ suspended over a trench of width $L$ are characterized by ${ }^{16,25}$ :

$$
\begin{gathered}
\lambda^{4}=\frac{4 \pi^{2} v(t L)^{2}}{3\left(1-v^{2}\right) \varepsilon} \\
A^{2}=\left[\frac{16 \varepsilon v}{3 \pi^{2}\left(1-v^{2}\right)}\right]^{1 / 2} t L
\end{gathered}
$$

Here, $\lambda$ and $A$ are the wavelength and amplitude of the rippling mode developed, and $\varepsilon$ is the compressive strain along the trench edges.
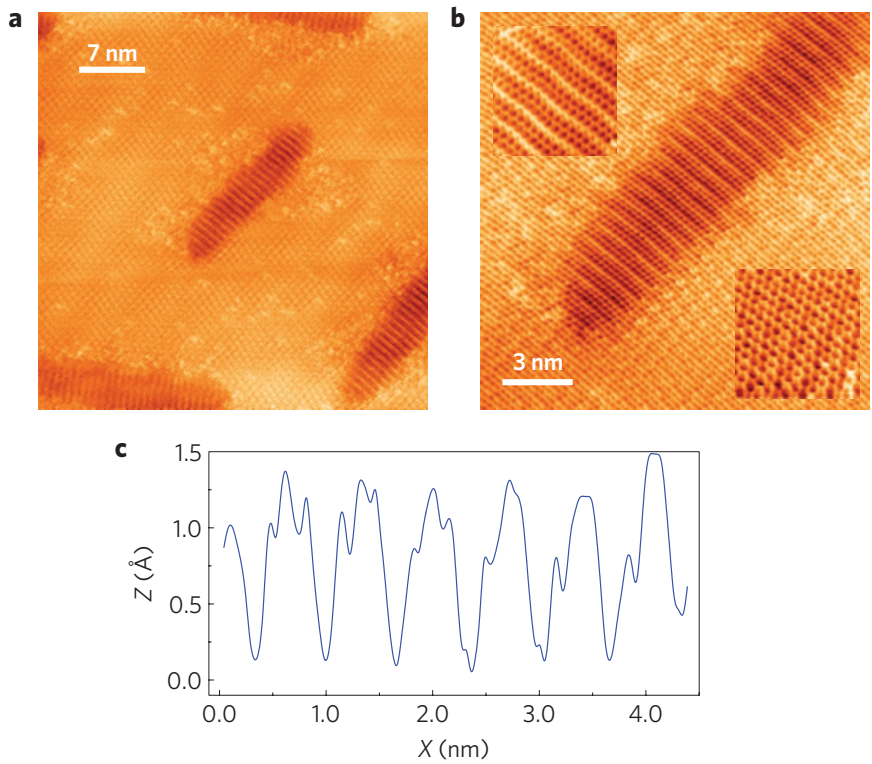

Figure 2 | Atomic-resolution STM images of graphene nanoripples.

a, STM image showing several nanotrenches of different orientations, all exhibiting graphene nanoripples over the trenches with the ripple crests always perpendicular to the trench edges. On the flat regions between the trenches, a Moiré pattern can be observed. b. Atomic-resolution STM image of a nanotrench exhibiting subnanometre graphene ripples. The magnified insets exhibit the honeycomb graphene lattice both over the flat substrate (bottom right) and the rippled region over the trenches (top left). c, Cross-section of the graphene ripples exhibiting a wavelength of $0.7 \mathrm{~nm}$, and an amplitude of $0.05 \mathrm{~nm}$. The extra corrugation of the line can be attributed to the positions of $\mathrm{C}$ atoms.

Although derived and validated at the macroscopic scale, the above formulae have been successfully applied to describe the rippling of suspended (few-layer) graphene sheets at microscopic wavelengths $(\lambda>300 \mathrm{~nm}$; ref. 6). Compelled to apply them to the monolayer, one immediately faces the ambiguity of defining the thickness $t$ for the single layer of $\mathrm{C}$ atoms. One approach ${ }^{26}$ is to assign $t=3.35 \AA$, that is, the experimentally measured interlayer spacing in graphite. Using parameter values of $L=5 \mathrm{~nm}$ (membrane width) and $\varepsilon=2 \%$, matching our experiments, together with the in-plane Poisson's ratio $(v=0.16)$, these formulae give a wavelength of $\lambda_{\text {theor }}=4.2 \mathrm{~nm}$ and an amplitude of $A_{\text {theor }}=2.6 \AA$. This is in striking contrast with our measurements $\left(\lambda_{\exp }=0.7 \mathrm{~nm}, A_{\exp }=0.5 \AA\right)$. Varying the strain between $0.5 \%$ and $5 \%$ to account for the experimental uncertainty does not significantly improve the agreement. Another approach is to base our modelling on a plate with effective thickness $t=0.8 \AA$, derived from atomistically computed in-plane $\left(26.6 \mathrm{eV}^{-2}\right)$ and bending $(1.6 \mathrm{eV}$ per atom) constants of the monolayer ${ }^{7,27}$. This second model predicts a very different rippling mode, of wavelength $\lambda_{\text {theor }}=2 \mathrm{~nm}$ and amplitude $A_{\text {theor }}=1.3 \AA$, which is still in obvious contrast with the experimental findings.

Besides the values of the measured individual quantities $(\lambda, A)$, we have also investigated the validity of the continuum mechanical equations relating them. A particularly useful relation to verify this can be established by combining equations (1a) and (1b). This way one can eliminate the strain (which cannot be accurately measured) and end up with: $A \lambda / L=\sqrt{8 v /\left(3\left(1-v^{2}\right)\right)} t$. When supplying the corresponding parameter values $(A=0.05 \mathrm{~nm}, \lambda=0.7 \mathrm{~nm}, L=5 \mathrm{~nm}, v=0.16$, $t=0.335 \mathrm{~nm}$ ) the above relation clearly fails by an order of magnitude even for $t=0.8 \AA$. 


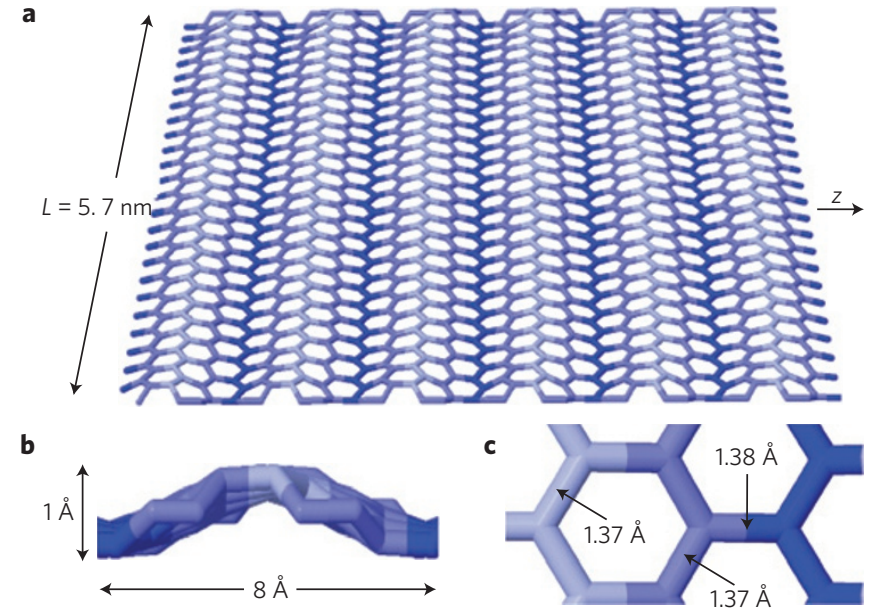

Figure 3 | Atomic-scale simulations of graphene nanoripples.

a,b Simulation of a 5.7-nm-wide infinitely long graphene nanoribbon subjected to $5 \%$ in-plane compression, revealing a periodic rippling morphology (a) of $0.8 \mathrm{~nm}$ wavelength and $1 \AA$ modulation (b). c, Details of the $\mathrm{C}-\mathrm{C}$ bond lengths; the colour scale indicates the height profile with light (dark) blue atoms at $0.5(-0.5) \AA$ heights.

The inability of the phenomenological continuum model to describe the peculiar subnanometre rippling observed in our experiments prompted us to perform accurate microscopic-level simulations. We studied the rippling of freestanding graphene nanoribbons with fixed armchair edges by means of conjugate gradient relaxation simulations and a density-functional-based tight-binding interatomic potential ${ }^{7,27}$, which describes explicitly the $\sigma$ and $\pi$ C-C bonding of graphene. To closely mimic the boundary conditions with a reduced number of atoms, the simulation domain containing 600 atoms was placed under periodic boundary conditions along the $z$ dimension, which corresponds to the nanotrench long dimension. The trench width dimension was modelled explicitly as the distance between the two strained dimer lines located at the edges measuring $L=5.7 \mathrm{~nm}$. For further technical details of the simulation method, see Supplementary Information. In contrast to the continuum model, we found that the microscopic approach can regain subnanometre rippling and thus confirms our interpretation of the STM observations. Indeed, Fig. 3a shows the periodic rippling exhibited by a graphene ribbon subjected to $5 \%$ edge biaxial compression. This metastable rippling mode presents an energy advantage of $62 \mathrm{meV}$ per atom over the compressed planar morphology. Its wavelength of $0.8 \mathrm{~nm}$ and amplitude of $0.5 \AA$ (Fig. $3 \mathrm{~b}$ ) are both in excellent agreement with our experimental results.

The striking contrast with the $t=3.35 \AA$ plate-based model predictions is a manifestation of the breakdown of the plate phenomenology ${ }^{7}$. In the monolayer, the resistance to bending involves the $\pi$-orbital misalignment between adjacent pairs of $\mathrm{C}$ atoms, and can be completely decoupled from its in-plane $\sigma$ bond deformations. Thus, an atomic membrane with such stiff in-plane bonding as the $\sigma$ bonds of graphene can ripple at the subnanometre scale, which implies almost no resistance against out-of-plane deformations. This behaviour is in sharp contrast with the phenomenology captured by the classical continuum plate, where the bending of a plate always induces the in-plane stretching and compression on the opposite sides of a neutral curved surface. Nevertheless, the malfunctioning of the continuum model goes beyond the issue of selecting an appropriate plate thickness. The failure of the $t=0.8 \AA$ second plate model, which formally gives a bending constant in agreement with the above microscopic

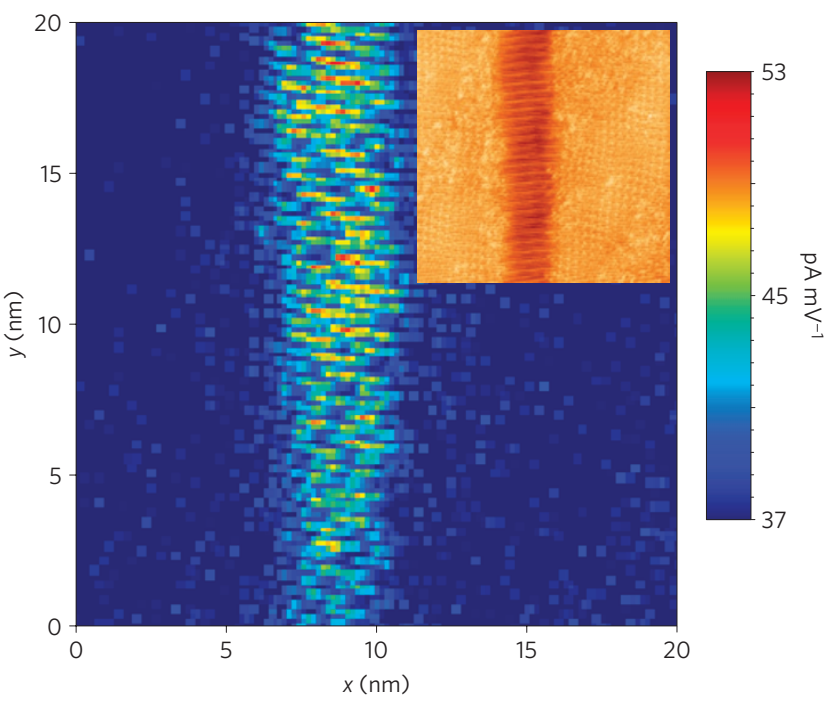

Figure 4 | Local electronic density of states map of graphene nanoripples. Spatially resolved differential tunnelling conductivity map (plotted at $U_{\text {bias }}=48 \mathrm{mV}$ ) exhibiting the periodic modulation of the local density of states on the graphene nanoripples. The inset shows the corresponding topographic image.

model, can be understood on the basis of the bond-length analysis shown in Fig. 3c. Recall that in both the equilibrium flat and inextensional bending case, the $\mathrm{C}-\mathrm{C}$ bonds measure $1.42 \AA$. In the relaxation through subnanometre rippling, the $\mathrm{C}-\mathrm{C}$ bonds still store a significant amount $(\sim 3.5 \%)$ of compressive strain. Therefore, the breakdown of the plate phenomenology allows the violation of the inextensible deformation assumption, which is key for deriving equations (1) (ref. 16). Of course, the experimental mechanical constraints set by the nanotrenches do not prohibit the onset of rippling modes in which bending would be accomplished in an approximately inextensible manner.

As the atomistic mechanisms allow a much easier out-of-plane deformation of graphene at the nanoscale as compared with classical plates, this ultrasoft bending behaviour might also be at the origin of the ultrastrong adhesion of the monolayers to different substrates ${ }^{28}$, by allowing a substantially better conformation to the substrate irregularities. In comparison, for multilayers, the interlayer coupling re-establishes the coupling between bending and in-plane deformations ${ }^{7}$, abruptly increasing the flexural rigidity ${ }^{29}$.

We have also performed spatially resolved tunnelling spectroscopy measurements $s^{2,10,12}$ to verify how the nanoscale structural ripples affect the local electronic properties of graphene $e^{9,30}$. In this measurement mode, individual tunnelling $I-V$ characteristics are acquired with the tip fixed over a specific location of the sample and the sample voltage ramped within a given window while measuring the corresponding tunnel current (for further details, see Supplementary Information). Figure 4 shows the differential tunnelling conductivity ( local density of electronic states) map obtained by plotting the numerical derivative of the individual current-voltage characteristics at each location for a sample bias of $U_{\text {bias }}=48 \mathrm{mV}$. The spectroscopy map reveals the periodic modulation of the local density of electronic states on the nanorippled region. This provides evidence that nanoscale structural ripples substantially affect the local electronic structure of graphene, giving rise to one-dimensional electronic superlattices.

The ability of graphene to ripple down to subnanometre wavelengths can be exploited in strain-engineering graphene-based nanoelectronic and nanoelectromechanical devices beyond the boundaries set by continuum mechanics. 
Received 1 April 2012; accepted 12 July 2012; published online 19 August 2012

\section{References}

1. Katsnelson, M. I. \& Geim, A. K. Electron scattering on microscopic corrugations in graphene. Phil. Trans. R. Soc. A 366, 195-204 (2008).

2. Vazquez de Parga, A. L. et al. Periodically rippled graphene: Growth and spatially resolved electronic structure. Phys. Rev. Lett. 100, 056807 (2008).

3. de Juan, F., Cortijo, A., Vozmediano, M. A. H. \& Cano, A. Aharonov-Bhom interferences from local deformations in graphene. Nature Phys. 7, 810-815 (2011).

4. Levy, N. et al. Strain-induced pseudo-magnetic fields greater than 300 Tesla in graphene nanobubbles. Science 329, 544-547 (2010).

5. Ni, G. X. et al. Quasi periodic nanoripples in graphene grown by chemical vapor deposition and its impact on charge transport. ACS Nano 6, 1158-1164 (2012).

6. Bao, W. et al. Controlled ripple texturing of suspended graphene and ultrathin graphite membranes. Nature Nanotech. 4, 562-566 (2009).

7. Zhang, D. B., Akatyeva, E. \& Dumitrica, T. Bending ultrathin graphene at the margins of continuum mechanics. Phys. Rev. Lett. 106, 255503 (2011).

8. Kim, E. \& Castro-Neto, A. H. Graphene as an electronic membrane. Europhys. Lett. 84, 57007 (2008).

9. Isacsson, A., Jonsson, L. M., Kinaret, J. M. \& Jonson, M. Electronic superlattices in corrugated graphene. Phys. Rev. B 77, 035423 (2008).

10. Teague, M. L. et al. Evidence of strain induced local conductance modulations in single-layer graphene on $\mathrm{SiO}_{2}$. Nano Lett. 9, 2542-2548 (2009).

11. Fasolino, A., Los, J. H. \& Katsnelson, M. I. Intrinsic ripples in graphene. Nature Mater. 6, 858-861 (2007)

12. Deshpande, A., Bao, W, Miao, F., Lau, C. N. \& LeRoy, B. J. Spatially resolved spectroscopy of monolayer graphene on $\mathrm{SiO}_{2}$. Phys. Rev. B 79, 205411 (2009).

13. Brey, L. \& Palacios, J. J. Exchange-induced charge inhomogeneities in rippled neutral graphene. Phys. Rev. B. 77, 041403(R) (2008).

14. Park, C. H., Yang, L., Son, Y. W., Cohen, M. L. \& Louie, S. G. Anisotropic behavior of massless Dirac fermions in graphene under periodic potentials. Nature Phys. 4, 213-217 (2008).

15. Boukhvalov, D. W \& Kastnelson, M. I. Enhancement of chemical activity in corrugated graphene. J. Chem. Phys. C 113, 14176-14168 (2009).

16. Cerda, E. \& Mahadevan, L. Geometry and physics of wrinkling. Phys. Rev. Lett. 90, 074302 (2003).

17. Pai, W. W. et al. Evolution of two-dimensional wormlike nanoclusters on metal surfaces. Phys. Rev. Lett. 86, 3088-3091 (2001).

18. Rasool, H. I. et al. Atomic-scale characterization of graphene on copper (100) single crystals. J. Am. Chem. Soc. 133, 12536-12543 (2011).

19. Rutter, G. M. et al. Scattering and interference in epitaxial graphene. Science 317, 219-222 (2007).

20. Tapaszto, L., Dobrik, G., Lambin, P. \& Biro, L. P. Tailoring the atomic structure of graphene nanoribbons by scanning tunneling microscope lithography. Nature Nanotech. 3, 397-401 (2008).
21. Hwang, C. et al. Initial stage of graphene growth on a Cu substrate. J. Phys, Chem. C 115, 22369-22374 (2011).

22. Wofford, J. M., Nie, S, McCarty, K. F., Bartelt, N. C. \& Dubon, O. D. Graphene islands on $\mathrm{Cu}$ foils: The interplay between shape, orientation, and defects. Nano Lett. 10, 4890-4896 (2010).

23. Chen, C. C. et al. Raman spectroscopy of ripple formation in suspended graphene. Nano Lett. 9, 4172-4176 (2009).

24. Zakharenko, K. V., Katsnelson, M. I. \& Fasolino, A. Finite temperature lattice properties of graphene beyond the quasiharmonic approximation. Phys. Rev. Lett. 102, 046808 (2009).

25. Wang, Z. \& Devel, M. Periodic ripples in suspended graphene. Phys. Rev. B 83, 125422 (2011).

26. Tsoukleri, G. et al. Subjecting a graphene monolayer to tension and compression. Small 5, 2397-2402 (2009).

27. Frauenheim, D., Köhler, T., Seifert, Th. \& Kaschner, G. Construction of tight-binding-like potentials on the basis of density-functional theory: Application to carbon. Phys. Rev. B 51, 12947-12957 (1995).

28. Koenig, S. P., Bodetti, N. G., Dunn, M. L. \& Bunch, J. S. Ultrastrong adhesion of graphene membranes. Nature Nanotech. 6, 543-546 (2011).

29. Koshkinen, P. \& Kit, O. O. Approximate modeling of spherical membranes. Phys. Rev. B 82, 235420 (2010).

30. Xu, K., Cao, P. \& Heath, J. R. Scanning tunneling microscopy characterization of the electrical properties of wrinkles in exfoliated graphene mono layers. Nano Lett. 9, 4446-4451 (2009).

\section{Acknowledgements}

The experimental work has been conducted within the framework of the Korean-Hungarian Joint Laboratory for Nanosciences through the Converging Research Center Program (2010K000980). L.T. acknowledges OTKA grant PD 91160 and the Bolyai fellowship and is grateful to the Alexander von Humboldt Foundation. P.N-I. and L.P.B. acknowledge OTKA grant K 101599. T.D. acknowledges NSF CAREER Grant CMMI-0747684

\section{Author contributions}

L.T. conceived and designed the experiments. L.T. and P.N-I. performed the STM experiments. S.J.K. and C.H. performed the growth experiments. T.D. provided the simulation results. L.T., T.D. and L.P.B. analysed the data. L.T. and T.D. wrote the paper. All of the authors discussed the results and commented on the manuscript.

\section{Additional information}

Supplementary information is available in the online version of the paper. Reprints and permissions information is available online at www.nature.com/reprints. Correspondence and requests for materials should be addressed to L.T.

\section{Competing financial interests}

The authors declare no competing financial interests. 